PREPARED FOR THE U.S. DEPARTMENT OF ENERGY, UNDER CONTRACT DE-AC02-76CH03073

PPPL-3495

PPPL-3495

UC-70

Shear-Alfvén Waves in Gyrokinetic Plasmas

by

W.W. Lee, J.L.V. Lewandowski, T.S. Hahm, and Z. Lin

October 2000
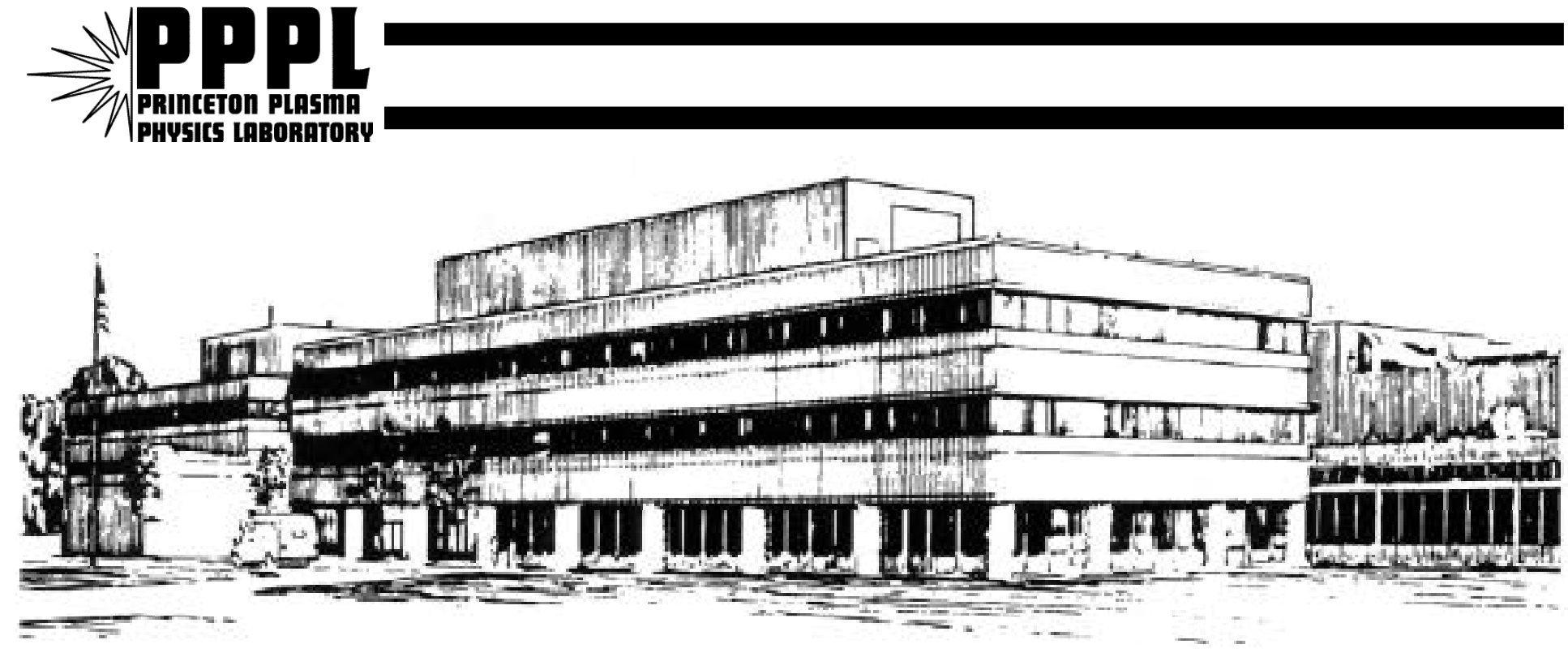

PRINCETON PLASMA PHYSICS LABORATORY PRINCETON UNIVERSITY, PRINCETON, NEW JERSEY 


\section{PPPL Reports Disclaimer}

This report was prepared as an account of work sponsored by an agency of the United States Government. Neither the United States Government nor any agency thereof, nor any of their employees, makes any warranty, express or implied, or assumes any legal liability or responsibility for the accuracy, completeness, or usefulness of any information, apparatus, product, or process disclosed, or represents that its use would not infringe privately owned rights. Reference herein to any specific commercial product, process, or service by trade name, trademark, manufacturer, or otherwise, does not necessarily constitute or imply its endorsement, recommendation, or favoring by the United States Government or any agency thereof. The views and opinions of authors expressed herein do not necessarily state or reflect those of the United States Government or any agency thereof.

\section{Availability}

This report is posted on the U.S. Department of Energy's Princeton Plasma Physics Laboratory Publications and Reports web site in Calendar Year 2000. The home page for PPPL Reports and Publications is: http://www.pppl.gov/pub_report/

DOE and DOE Contractors can obtain copies of this report from:

U.S. Department of Energy

Office of Scientific and Technical Information

DOE Technical Information Services (DTIS)

P.O. Box 62

Oak Ridge, TN 37831

Telephone: (865) 576-8401

Fax: (865) 576-5728

Email: reports@adonis.osti.gov

This report is available to the general public from:

National Technical Information Service

U.S. Department of Commerce

5285 Port Royal Road

Springfield, VA 22161

Telephone: $1-800-553-6847$ or

(703) $605-6000$

Fax: (703) 321-8547

Internet: http://www.ntis.gov/ordering.htm 


\title{
Shear-Alfven Waves in Gyrokinetic Plasmas
}

\author{
W. W. Lee, J. L. V. Lewandowski, T. S. Hahm, and Z. Lin \\ Plasma Physics Laboratory, Princeton University, Princeton, NJ 08543
}

\begin{abstract}
It is found that the thermal fluctuation level of the shear-Alfven waves in a gyrokinetic plasma decreases with plasma $\beta\left(\equiv c_{s}^{2} / v_{A}^{2}\right)$, where $c_{s}$ is the ion acoustic speed and $v_{A}$ is the Alfven velocity. This unique thermodynamic property based on the fluctuation-dissipation theorem is verified in this paper using a new gyrokinetic particle simulation scheme, which splits the particle distribution function into the equilibrium part as well as the adiabatic and nonadiabatic parts.
\end{abstract}

PACS numbers: 52.25.Gj, 52.35.Hr, 52.65.Rr, 52.65.Tt 
Gyrokinetic particle simulation [1,2] was invented for the purpose of reducing the temporal and spatial disparities in the simulation plasma when one is only interested in the long-wavelength and low-frequency modes in magnetically confined plasmas. Another benefit of the gyrokinetic approach is the reduction of the numerical noise in the simulation $[2,3]$. On the other hand, the perturbative $(\delta f)$ particle simulation scheme [4] has made the numerical noise more or less a non-issue, with the argument that one can always make the numerical noise arbitrarily small [5]. In this paper, we will show that the noise issue is still relevant for perturbative gyrokinetic particle simulation when finite- $\beta$ effects associated with shear-Alfven physics are important. Both analytic and numerical results will be presented. The former is based on the usual fluctuation-dissipation theorem and the latter uses a new simulation scheme which is the finite- $\beta$ extension of the split-weight perturbative gyrokinetic particle simulation scheme in the electrostatic approximation [6]. Specifically, the new split-weight scheme breaks up the distribution function into an equilibrium part, $F_{0}$, as well as an adiabatic part and a non-adiabatic part. The adiabatic part is associated with the product of $F_{0}$ and the effective potential of $\psi=\phi+\int \partial A_{\|} / c \partial t d x_{\|}$, and the nonadiabatic part is followed dynamically. Here, $\phi$ is the electrostatic potential, $A_{\|}$is the vector potential, and $x_{\|}$is the spatial coordinate along the field-line. As we will discuss, without the use of $\psi$, numerical noise can interfere with the formation of shear-Alfven normal modes.

In the gyrokinetic units of $\rho_{s}\left(\equiv \sqrt{\tau} \rho_{i}\right)$ and $\Omega_{i}^{-1}$ for length and time, respectively, the governing gyrokinetic Vlasov equation for a finite- $\beta$ plasma in slab geometry in the limit of $k_{\perp}^{2} \rho_{i}^{2} \ll 1$ can be written as [7]

$$
\frac{d F_{\alpha}}{d t} \equiv \frac{\partial F_{\alpha}}{\partial t}+v_{\|} \hat{\mathbf{b}} \cdot \frac{\partial F_{\alpha}}{\partial \mathbf{x}}-\nabla \phi \times \hat{\mathbf{b}}_{0} \cdot \frac{\partial F_{\alpha}}{\partial \mathbf{x}}+s_{\alpha} v_{t \alpha}^{2} \frac{\partial \psi}{\partial x_{\|}} \frac{\partial F_{\alpha}}{\partial v_{\|}}=0,
$$

where $\tau \equiv T_{e} / T_{i}, \alpha$ denotes species, $v_{t e}^{2}=m_{i} / m_{e}, v_{t i}^{2}=1 / \tau, s_{e}=1, s_{i}=-\tau$,

$$
\begin{gathered}
\hat{\mathbf{b}} \equiv \hat{\mathbf{b}}_{0}+\frac{\delta \mathbf{B}}{B_{0}}=\frac{\mathbf{B}_{\mathbf{0}}}{B_{0}}+\nabla A_{\|} \times \hat{\mathbf{b}}_{0}, \\
\mathbf{E}^{L}=-\nabla \phi, \quad E_{\|}^{T}=-\frac{\partial A_{\|}}{\partial t},
\end{gathered}
$$


and the superscripts $L$ (ongitudinal) and $T$ (ransverse) denote the decomposition relative to the direction of wave propagation. The effective potential in Eq. (1) is defined as

$$
\frac{\partial \psi}{\partial x_{\|}} \equiv-\left(\mathbf{E}^{L}+E_{\|}^{T} \hat{\mathbf{b}}_{0}\right) \cdot \hat{\mathbf{b}}=\frac{\partial \phi}{\partial x_{\|}}+\frac{\partial A_{\|}}{\partial t},
$$

and $\partial / \partial x_{\|} \equiv \hat{\mathbf{b}} \cdot \nabla$. The gyrokinetic Poisson's equation for $k_{\perp}^{2} \rho_{i}^{2} \ll 1$ can be simplified as

$$
\nabla_{\perp}^{2} \phi=\int\left(F_{e}-F_{i}\right) d v_{\|}
$$

where the electrostatic potential $\phi$ is normalized by $T_{e} / e$ and $\int F_{0 \alpha} d v_{\|}=1$. Ampere's law then becomes

$$
\nabla_{\perp}^{2} A_{\|}=\beta \int v_{\|}\left(F_{e}-F_{i}\right) d v_{\|}
$$

where the vector potential $A_{\|}$is normalized by $c T_{e} / e c_{s}, \beta \equiv c_{s}^{2} / v_{A}^{2}, v_{A} \equiv c \lambda_{D} / \rho_{s}$ is the Alfven speed, and $\lambda_{D}$ is the electron Debye length. [Note that the ion acoustic speed $c_{s}\left(\equiv \rho_{s} \Omega_{i}\right)$ is unity in the gyrokinetic unit.] Equations (1)-(6) are the so-called (electromagnetic) Darwin model, in which the transverse induction electric current, $\partial \mathbf{E}^{T} / \partial t$, is neglected in Ampere's law. The approximation of $J_{\|}^{T} \approx J_{\|}$valid for $k_{\|} \ll k_{\perp}$ is also used here. To expedite the calculation of $\partial \psi / \partial x_{\|}$of Eq. (4), a generalized Ohm's law in shearless slab geometry, by combining Eqs. (1) - (6), can be written as

$$
\begin{gathered}
{\left[\nabla_{\perp}^{2}-\beta\left(\frac{m_{i}}{m_{e}} \int F_{e} d v_{\|}+\int F_{i} d v_{\|}\right)\right] \frac{\partial \psi}{\partial x_{\|}}=\beta \frac{\partial}{\partial x_{\|}} \int v_{\|}^{2}\left(F_{i}-F_{e}\right) d v_{\|}} \\
-\beta \nabla \phi \times \hat{\mathbf{b}}_{0} \cdot \nabla \int v_{\|}\left(F_{i}-F_{e}\right) d v_{\|}-\frac{\partial}{\partial x_{\|}} \int\left(F_{i}-F_{e}\right) d v_{\|} .
\end{gathered}
$$

Starting from Eq. (1) and using

$$
F_{\alpha}=F_{0 \alpha}+\delta f_{\alpha}
$$

and $\partial F_{0 \alpha} / \partial t+v_{\|} \hat{\mathbf{b}}_{0} \cdot \partial F_{0 \alpha} / \partial \mathbf{x}=0$, we obtain

$$
\frac{d \delta f_{\alpha}}{d t}=-\frac{d F_{0 \alpha}}{d t}=s_{\alpha} v_{\|} \frac{\partial \psi}{\partial x_{\|}} F_{0 \alpha},
$$


where $d / d t$ is defined in Eq. (1) and the zeroth-order inhomogeneity is ignored. The generalized Ohm's law, Eq. (7), should then be changed accordingly as

$$
\left[\nabla_{\perp}^{2}-\beta \frac{m_{i}}{m_{e}}\right] \frac{\partial \psi}{\partial x_{\|}}=\beta \frac{\partial}{\partial x_{\|}} \int v_{\|}^{2}\left(\delta f_{i}-\delta f_{e}\right) d v_{\|}-\frac{\partial}{\partial x_{\|}} \int\left(\delta f_{i}-\delta f_{e}\right) d v_{\|},
$$

where the higher order nonlinear terms have been dropped.

The theoretical and numerical properties of the shear-Alfven waves can be studied as follows. The linearized version of Eq. (8),

$$
\frac{\partial \delta f_{\alpha}}{\partial t}+v_{\|} \frac{\partial \delta f_{\alpha}}{\partial x_{\|}}=s_{\alpha} v_{\|} \frac{\partial \psi}{\partial x_{\|}} F_{0 \alpha}
$$

with the ansatz of $\exp \left(i k_{\|} x_{\|}-i \omega t\right)$ gives

$$
\delta f_{\alpha}=\left(1+\frac{\omega}{k_{\|} v_{\|}-\omega}\right) s_{\alpha} F_{0 \alpha} \psi
$$

Substituting it into Eq. (9) and assuming cold ion response of $\omega / k_{\|} \gg v_{t i}$, we obtain the dispersion relation as

$$
D \equiv \frac{1}{k^{2} \lambda_{D}^{2}}\left[k_{\perp}^{2}+\left(1-\beta \frac{\omega^{2}}{k_{\|}^{2}}\right)\left(1+X_{e}\right)\right]=0
$$

where $D$ is the dielectric constant for $k^{2} \lambda_{D}^{2} \ll 1$ and

$$
X_{\alpha} \equiv \frac{\omega}{\sqrt{2} k_{\|} v_{t \alpha}} Z\left(\frac{\omega}{\sqrt{2} k_{\|} v_{t \alpha}}\right)
$$

For the cold electron response, $\omega \gg k_{\|} v_{t e}$, Eq. (12) gives the normal modes as

$$
\omega= \pm \frac{\omega_{H}}{\sqrt{1+\omega_{p e}^{2} / c^{2} k^{2}}}
$$

where $c / \omega_{p e}\left(\equiv \rho_{s} \sqrt{m_{e} / m_{i} \beta}\right)$ is the electron skin depth. In the electrostatic limit of $\omega_{p e}^{2} / c^{2} k^{2} \rightarrow 0$, it recovers the shear-Alfven modes in the electrostatic limit, i.e., $\omega_{H}[\equiv$ $\left.\left(k_{\|} / k_{\perp}\right) \sqrt{m_{i} / m_{e}} \Omega_{i}\right]$ as discussed in Ref. [2]. Equation (13), valid for $\beta \ll m_{e} / m_{i}$, shows that the magnitude of the shear-Alfven frequencies becomes lower for higher $\beta$ 's. For the warm electron response of $\omega \ll k_{\|} v_{t e}$, the oscillation frequencies from Eq. (12) become

$$
\omega= \pm k_{\|} v_{A} \sqrt{1+k_{\perp}^{2}}
$$


and the corresponding damping rate is

$$
\frac{\gamma}{\omega}=-\frac{1}{2} \sqrt{\frac{\pi}{2}} \frac{\omega_{l}}{k_{\|} v_{t e}} \frac{k_{\perp}^{2}}{1+k_{\perp}^{2}} .
$$

where $v_{A} \equiv \sqrt{1 / \beta}$ is the Alfven velocity. These are the kinetic shear-Alfven waves for $\beta \gg m_{e} / m_{i}$ and both the magnitude of the frequency and the damping rate continue to decrease for higher $\beta$ 's. For $\beta=1$, the normal modes are $\omega= \pm k_{\|} c_{s} \sqrt{1+k_{\perp}^{2}}$.

The corresponding fluctuation levels for these modes can be derived as follows. Starting from the fluctuation-dissipation theorem [10] for a system with $\tau=1$, we can express the thermal fluctuation level as

$$
V k^{2}|\psi(\mathbf{k})|^{2} / 8 \pi=\frac{T_{e}}{2} \sum_{\Omega} \frac{1}{\left|\omega \partial D_{R} / \partial \omega\right|_{\Omega}}
$$

where $D_{R}$ is the real part of the dielectric constant, Eq. (12), and $\Omega$ is the normal mode and $V$ is the volume of the system. For the cold electron response of $\omega \gg k_{\|} v_{t e}$, we obtain

$$
V k^{2}|\psi(\mathbf{k})|^{2} / 8 \pi=\frac{T_{e}}{2} \frac{\lambda_{D}^{2}}{\rho_{s}^{2}} \frac{1}{1+\omega_{p e}^{2} / c^{2} k^{2}},
$$

and, correspondingly,

$$
\left|\frac{e \psi}{T_{e}}\right|=\frac{1}{\sqrt{N} k \rho_{s}} \frac{1}{\sqrt{1+\omega_{p e}^{2} / c^{2} k^{2}}},
$$

where $N$ is the number of simulation particles in the waves of interest. Apparently, there is reduction in the noise level as shown in Eq. (18) due to finite- $\beta$ effects. Equations (17) and (18) are valid for $\beta \ll m_{e} / m_{i}$. In the limit of $\beta=0(\psi \rightarrow \phi)$, we recover the level of $\left|e \phi / T_{e}\right|=1 / \sqrt{N} k \rho_{s}$ as given by Ref. [2,3]. For the warm electron response of $\omega \ll k_{\|} v_{t e}$, Eq. (16) gives

$$
V k^{2}|\psi(\mathbf{k})|^{2} / 8 \pi=\frac{T_{e}}{2} \frac{k^{2} \lambda_{D}^{2}}{1+k^{2} \rho_{s}^{2}},
$$

and, in turn, the noise level becomes

$$
\left|\frac{e \psi}{T_{e}}\right|=\frac{1}{\sqrt{N}} \frac{1}{\sqrt{1+k^{2} \rho_{s}^{2}}}
$$


Thus, Eqs. (19) and (20) indicate that, for $\beta \gg m_{e} / m_{i}$, the noise level is much reduced and is nearly constant for $k_{\perp}^{2} \rho_{s}^{2} \ll 1$. For $\beta=0$ with warm electrons and cold ions, Eq. (12) gives the normal mode of $\omega= \pm k_{\|} c_{s} / \sqrt{1+k^{2} \rho_{s}^{2}}$, which is the gyrokinetic version of the ion acoustic waves and its thermal energy and noise level are the same as those given in Eqs. (19) and (20) with $\psi$ replaced by $\phi$. For $k^{2} \rho_{s}^{2} \ll 1$, we recover the noise level for the usual ion acoustic waves.

The total fluctuation level in a finite- $\beta$ gyrokinetic plasma can be obtained by integrating over the the whole $\omega$ spectra [3], i.e.,

$$
V k^{2}|\psi(\mathbf{k})|^{2} / 8 \pi=\frac{T}{2}\left(\frac{1}{D(\omega=\infty)}-\frac{1}{D(\omega=0)}\right)=\frac{T_{e}}{2} \frac{\lambda_{D}^{2}}{\rho_{s}^{2}} .
$$

The corresponding fluctuation potential becomes

$$
\left|\frac{e \psi}{T_{e}}\right|=\frac{1}{\sqrt{N} k \rho_{s}}
$$

These levels are the same as those obtained earlier for finite- $\beta$ plasmas $[8,11]$ and are also the same as the electrostatic cases $[2,3]$. Thus, there is no reduction in the total noise level due to finite $\beta$ effects. However, as shown in Eqs. (17)-(20), noise reduction indeed exists, but only for the shear-Alfven normal modes. The difference comes from the fact that, in a gyrokinetic plasma, there is no damping mechanism to reduce the noise produced by the fast moving electrons.

The interest in utilizing the desirable numerical properties of shear-Alfven waves for simulation purposes was first mentioned in Ref. [12]. However, a simple application of the perturbative $\delta f$ scheme [4] to solve Eqs. (8) and (9) was limited to low $\beta$ plasmas, i.e., $\beta \leq m_{e} / m_{i}$. The subsequent work [9] using the canonical momentum as a phase space variable [7] encountered similar restrictions. In both cases, numerical noise was cited as a possible suspect - in agreement with our analyses presented here. Namely, the interference of the nonadiabatic responses of the fast electrons with the collective oscillations of shearAlfven waves is the culprit.

To prove this point numerically, we have devised a new simulation scheme, which is the 
extension of the split-weight $\delta f$ scheme [6] to finite- $\beta$ plasmas. Briefly, the scheme divides the perturbed electron distribution into the adiabatic and non-adiabatic parts, i.e.,

$$
\delta f_{e}=\psi F_{0 e}+\delta h_{e}
$$

From $d \psi / d t=\partial \psi / \partial t+v_{\|} \hat{\mathbf{b}} \cdot \partial \psi / \partial \mathbf{x}-\nabla \phi \times \hat{\mathbf{b}}_{0} \cdot \partial \psi / \partial \mathbf{x}$ and Eq. (1), and dropping the nonlinear terms, we obtain

$$
\frac{d \delta h_{e}}{d t}=-\frac{\partial \psi}{\partial t} F_{0 e}
$$

Defining

$$
w^{N A} \equiv \delta h_{e} / F_{e}
$$

the weight equation can then be written as

$$
\frac{d w^{N A}}{d t}=\frac{1}{F_{e}} \frac{d \delta h_{e}}{d t}=-\frac{1-w^{N A}}{1+\psi} \frac{\partial \psi}{\partial t}
$$

The corresponding equations of motion are

$$
\begin{gathered}
\frac{d \mathbf{x}}{d t}=v_{\|} \hat{\mathbf{b}}_{0}-\nabla\left(\phi-v_{\|} A_{\|}\right) \times \hat{\mathbf{b}}_{0}, \\
\frac{d v_{\|}}{d t}=s_{\alpha} v_{t \alpha}^{2} \frac{\partial \psi}{\partial x_{\|}},
\end{gathered}
$$

and the generalized Ohm's law is simplified as

$$
\left(\nabla_{\perp}^{2}-1\right) \frac{\partial \psi}{\partial x_{\|}}=\beta \frac{\partial}{\partial x_{\|}} \int v_{\|}^{2}\left(\delta f_{i}-\delta h_{e}\right) d v_{\|}-\frac{\partial}{\partial x_{\|}} \int\left(\delta f_{i}-\delta h_{e}\right) d v_{\|} .
$$

where the perturbed ion distribution, $\delta f_{i}$, can be calculated by the standard $\delta f$ scheme [4] and the nonadiabatic electron contribution is given by

$$
\delta h_{e}=\sum_{j=1}^{N} w_{j}^{N A} \delta\left(\mathbf{x}-\mathbf{x}_{j}\right) \delta\left(v_{\|}-v_{\| j}\right) .
$$

Taking $\partial / \partial t$ of Eqs. (9) and (27) and using Eqs. (8) and (23), we arrive at

$$
\left[\nabla_{\perp}^{2}-\beta \frac{m_{i}}{m_{e}}\right] \frac{\partial \psi}{\partial t}=-\beta \frac{\partial}{\partial x_{\|}} \int v_{\|}^{3}\left(\delta f_{i}-\delta h_{e}\right) d v_{\|}+\frac{\partial}{\partial x_{\|}} \int v_{\|}\left(\delta f_{i}-\delta h_{e}\right) d v_{\|} .
$$


Equations (24)-(28) form the basic set for finite- $\beta$ split-weight particle simulation.

The simulation has been carried out with a one dimensional simulation code, GK1D. In the code, both $x$ and $z$ are ignorable coordinates, $y=\theta x_{\|}$, and $\theta \ll 1$ is the angle between the external $B$ field and the $z$ axis. This is a linear simulation in which Eqs. (24) - (26) are simplified to $d x_{\|} / d t=v_{\|}, d v_{\|} / d t=0$, and $d w^{N A} / d t=-\partial \psi / \partial t$, respectively. The simulation uses a 64 grid system with 6765 particles and includes only the modes with $k \rho_{s} \approx \pm 0.4$. The other parameters are: $m_{i} / m_{e}=1837, \tau=1, \theta=0.01$, and $a$ (particle size) $=0$. In all the simulations reported here, we set the initial amplitude to $e \psi / T_{e}=100 \%$. In Fig. 1, the fluctuation (noise) levels and frequencies for the $\beta=0$ case are shown, where $\Omega_{i} \Delta t=0.2$ is the time step used in the simulation. The noise level of $e \psi / T_{e} \simeq 3 \%$ at $\Omega_{i} t=60$ agrees well with that given by Eq. (18). However, the noise fluctuates wildly in the ensuing simulation and reaches as high as $20 \%$ at times. This unphysically high amplitude of oscillations increases with larger time steps. Furthermore, the simulation frequencies shown in Fig. 1 are slightly higher than the theoretical values given by Eq. (13), probably due to the enhanced noise as well. The results for the case of $\beta=0.1 \%$ are shown in Fig. 2 , where $\Omega_{i} \Delta t=2$ is used. Both the linear frequencies and the damping rate of $(\omega+i \gamma) / \Omega_{i}= \pm 0.13-i 0.011$ are in good agreement with the theoretical predictions of Eqs. (14) and (15). In the steady state, the noise level improves to $e \psi / T_{e} \simeq 1.2 \%$ and also agrees with the amplitude predicted by Eq. (20) and there is no numerical enhancement. For $\beta=10 \%$ and $\Omega_{i} \Delta t=2$, the frequencies and the damping rate of $(\omega+i \gamma) / \Omega_{i}= \pm 0.014-i 0.00012$, as given by Fig. 3, are in excellent agreement with those from Eqs. (14) and (15). For the last two cases, the time step is restricted by the parallel transit time requirement of $k_{\|} v_{t e} \Delta t<1$ [2]. 

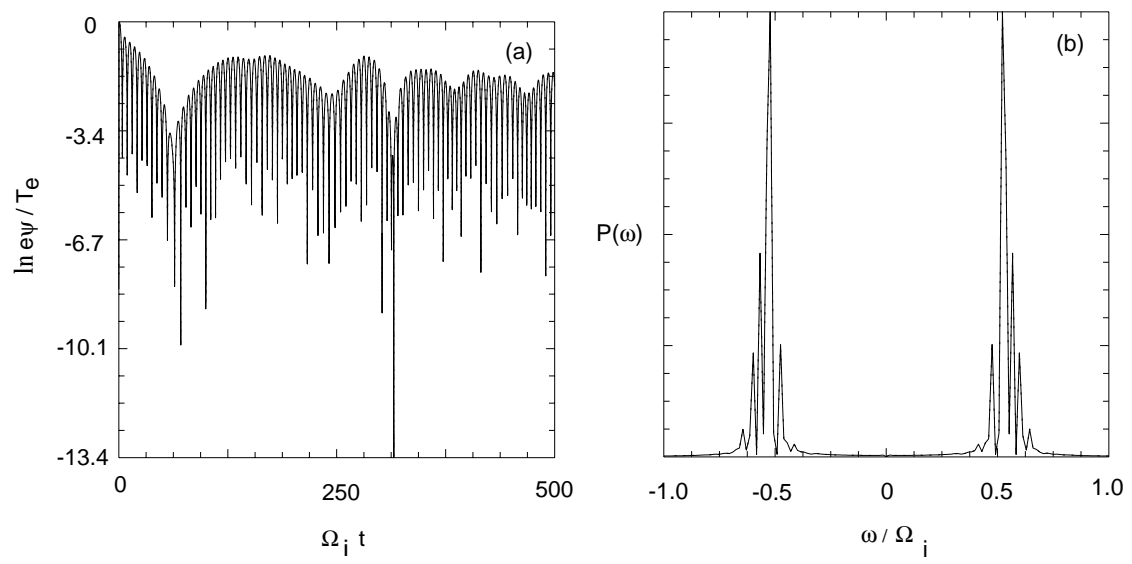

Figure 1. (a) Damping rate and noise level, and (b) frequencies for the shear-Alfven waves for $\beta=0.0 \%$
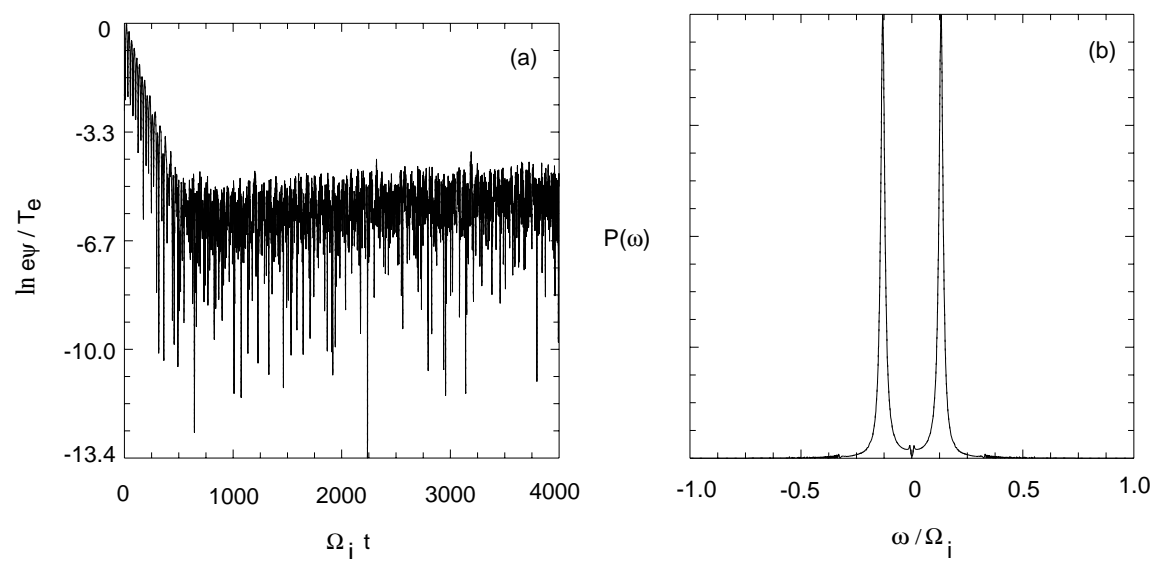

Figure 2. (a) Damping rate and noise level, and (b) frequencies for the shear-Alfven waves

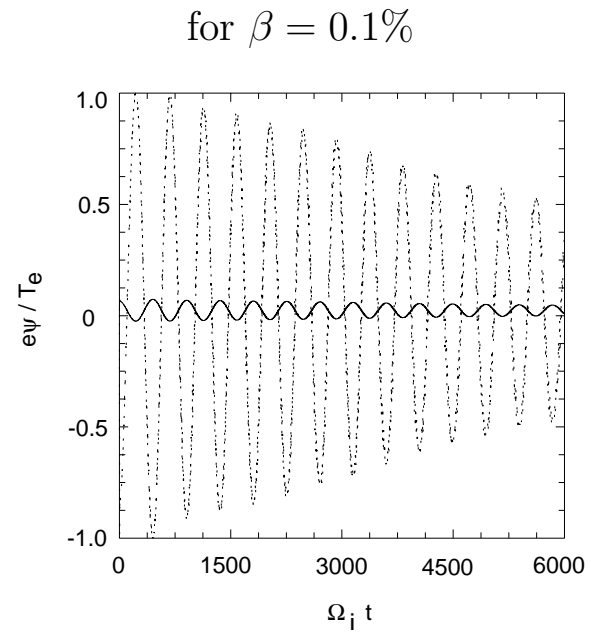

Figure 3. Shear-Alfven oscillations for $\beta=10.0 \%$

In conclusion, we believe that we have finally resolved a decade old puzzle concerning the 
favorable numerical trend for a finite- $\beta$ gyrokinetic plasma [12], which is found here to be intimately related to the unique thermodynamic feature of the shear-Alfven waves. Without such an understanding, one would conclude from Eq. $(22)[8,11]$ that finite- $\beta$ gyrokinetic particle simulation of long wavelength modes with $k \rho_{s} \rightarrow 0$ is nearly impossible. Most of all, we have developed a new simulation scheme that can be generalized to multi-dimensional simulations of finite- $\beta$ plasmas.

The research was supported by the Plasma Science Accelerated Computing Initiative of the U. S. DoE. 


\section{REFERENCES}

[1] W. W. Lee, Phys. Fluids 26, 556 (1983).

[2] W. W. Lee, J. Comput. Phys. 72, 243 (1987).

[3] J. A. Krommes, W. W. Lee and C. Oberman, Phys. Fluids 29, 2421 (1986).

[4] S. E. Parker and W. W. Lee, Phys. Fluids B 5, 77 (1993).

[5] G. Hu and J. A. Krommes, Phys. plasmas 1, 863 (1994).

[6] I. Manuilskiy and W. W. Lee, Phys. Plasmas 7, 1381 (2000).

[7] T. S. Hahm, W. W. Lee and A. Brizard, Phys. Fluids 31, 1940 (1987).

[8] J. V. W. Reynders, Ph.D. thesis, Princeton University (1992).

[9] J. C. Cummings, Ph.D. thesis, Princeton University (1995).

[10] Yu. L. Klimontovich, The statistical Theory of Non-Equilibrium Processes in a Plasma, (MIT Press, 1967).

[11] J. A. Krommes, Phys. Rev. Lett. 70, 3067 (1993); ibid. Phys. Fluids B 5, 2405 (1993); ibid. Phys. Fluids B 5, 1066 (1993).

[12] W. W. Lee, T. S. Hahm and J. V. W. Reynders, Bull. Am. Phys. Soc. 34, 2044 (1989). 
The Princeton Plasma Physics Laboratory is operated by Princeton University under contract with the U.S. Department of Energy.

\author{
Information Services \\ Princeton Plasma Physics Laboratory \\ P.O. Box 451 \\ Princeton, NJ 08543
}

Phone: 609-243-2750

Fax: 609-243-2751

e-mail: pppl_info@pppl.gov

Internet Address: http://www.pppl.gov 